\title{
Endovascular Management of Ectopic Variceal Bleeding after Hepaticojejunostomy
}

\author{
Amitha Vikrama K. S. ${ }^{1} \quad$ Yugandhar Samireddypalle \\ ${ }^{1}$ Department of Interventional Radiology, Sakra World Hospital, \\ Bangalore, Karnataka, India
}

J Clin Interv Radiol ISVIR 2019;3:69-70

A 62-year-old woman with a prior history of cholangiocarcinoma, left hepatectomy, hepaticojejunostomy, and portal vein reconstruction in September 2017 presented with recurring episodes of melena since March, 2018. Endoscopy revealed gastric varices, and the patient underwent multiple sessions of endoscopic management of the varices. However, the episodes of melena continued. Contrast-enhanced computed tomography (CT) of the abdomen revealed occlusion of the main portal vein with multiple venous collaterals at the hepaticojejunostomy ( - Fig. 1A, B). She was referred to the Interventional Radiology department for further management. On examination, she was clinically stable with pulse rate of 78 beats/min and blood pressure of 138/70 mm Hg. Her liver function tests, renal function tests, and coagulation parameters were normal. Hemoglobin was $12 \mathrm{gm} / \mathrm{dL}$. Portal vein recanalization followed by embolization of the varices was planned.

Percutaneous transhepatic access of a peripheral portal vein radicle of the segment VI was achieved using a micropuncture set (AccuStick, Boston Scientific). Portal venography revealed occlusion of the main portal vein and filling of left gastric vein ( - Fig. 1C). The occluded segment of the main portal vein was crossed using a 0.035 -in guidewire (Terumo). Balloon angioplasty of the occluded portal vein was performed using a $6-\times 40-\mathrm{mm}$ balloon. This was followed by placement of a $10-\mathrm{mm} \times 60-\mathrm{mm}$ self-expanding metallic stent (Absolute Pro; Abbott) ( - Fig. 2A, B). The stent was dilated using a $10-\times 40-\mathrm{mm}$ balloon. Postprocedure venography revealed satisfactory antegrade flow across the stent. There was filling of multiple venous collaterals at the hepaticojejunostomy from the jejunal tributaries of the superior mesenteric vein (-Fig. 2A). The left gastric vein was no longer visualized. These venous collaterals were superselectively catheterized, and embolization was performed using 33\% n-butyl-cyanoacrylate-Lipiodol mixture (-Fig. 2B). Postembolization venography revealed good antegrade flow across the stent with no filling of the varices at the

\author{
Chinmaya Deepak ${ }^{1}$
}

Address for correspondence Amitha Vikrama, KS, MD, DNB, FVIR, Department of Interventional Radiology, Sakra World Hospital, Bangalore 560103, Karnataka, India (e-mail: amithavikrama@gmail.com).

hepaticojejunostomy ( - Fig. 2C). The portal vein access tract was embolized using $n$-butyl-cyanoacrylate. There were no procedural or immediate postprocedural complications. The patient was discharged in a stable condition without any further complaints of melena. Follow-up Doppler at 1 week of the procedure revealed a patent portal vein with good hepatopetal flow.

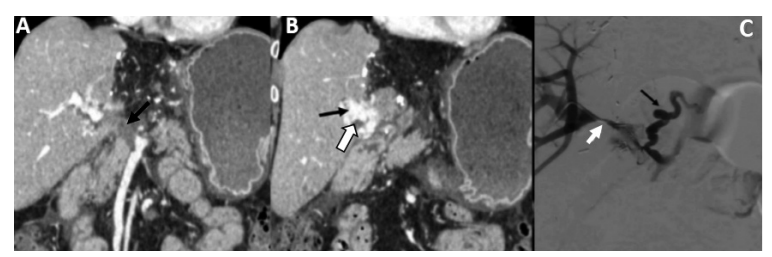

Fig. 1 (A) Coronal reformatted CT image at the level of hepatic hilum demonstrating portal vein thrombosis extending from the portosplenic confluence to the porta (arrow). The intrahepatic right branch of portal vein is patent. (B) Coronal reformatted CT image demonstrating multiple ectopic jejunal varices at the hepaticojejunostomy (black arrow). The hypodense thrombus within the portal vein is also noted (white arrow). (C) Portography demonstrating occlusion of the main portal vein (white arrow). There is prominent left gastric vein (black arrow) probably feeding the gastric collaterals.

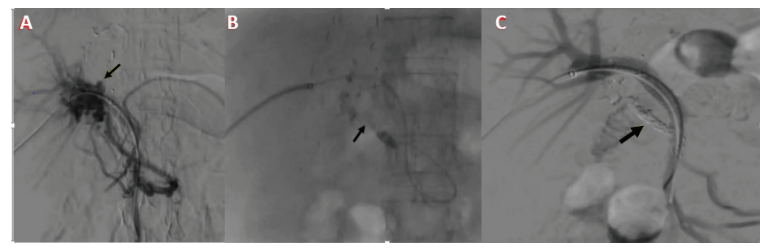

Fig. 2 (A) Poststenting portal venography demonstrating satisfactory antegrade flow across the portal vein. Multiple jejunal varices are seen supplied by the jejunal branches of the superior mesenteric vein (arrow). Note that the left gastric vein is not opacified. (B) Superselective embolization of the varices using 33\% n-butyl-cyanoacrylate (arrow). (C) Postembolization portography demonstrating good antegrade flow across the main portal vein without any filling of the varices. The glue cast of the embolization can be seen (arrow).

\section{received}

August 9, 2018

accepted after revision

November 13, 2018

published online

April 22, 2019
DOI https://doi.org/

$10.1055 / \mathrm{s}-0039-1681118$

ISSN 2457-0214.
C2019 by Indian Society of Vascular and Interventional Radiology
License terms

()(1) $\Theta \circledast$ 


\section{Discussion}

The incidence of ectopic varices at hepaticojejunostomy varies from 22 to $28 \%$ at 4 years following surgery for malignancy. ${ }^{1}$ The varices are secondary to localized portal hypertension from extrahepatic portal venous obstruction and can result in gastrointestinal bleeding. Extrahepatic portal venous obstruction after surgery may result in ectopic varices at multiple sites, including the stomach, duodenum, jejunum, ileum, and rectum. Sometimes, bleeding can be torrential and life threatening with poor prognosis. ${ }^{2}$ There are multiple treatment options, including surgery, endoscopic therapy, and interventional radiologic management. Conventional surgery aims at dissection of the hepaticojejunostomy site with re-anastomosis that results in disconnection of the jejunal varices. The risks of surgery include postoperative liver failure and recurrence of new varices. ${ }^{2}$ Surgical (mesocaval) shunt can also be used as a definitive surgical treatment. However, this shunt surgery has a risk for occlusion, the possibility of encephalopathy, and operative morbidities. ${ }^{3}$

Endoscopic management of these varices can be considered, but there is anatomic difficulty in reaching the afferent jejunal loop from the duodenum using the gastroduodenoscopy. Single-balloon endoscopy, double-balloon endoscopy, and adult variable stiffness colonoscopy have been used to reach these varices for clipping. Some have utilized push endoscopy using pediatric colonoscope and occluded the varices by direct injection of glue into the varices. ${ }^{4}$ In one case, percutaneous transhepatic cholangioscopy was performed for direct puncture of the varices and glue injection that had to be repeated several times. ${ }^{5}$

Interventional radiologic management of these varices has evolved as a safe and effective first-line therapy. Transjugular intrahepatic portosystemic shunt (TIPS) followed by portal vein stenting can be considered but is often technically difficult due to portal venous obstruction. In some cases, transhepatic or intraoperative portogra-phy followed by embolization of the varices was performed. ${ }^{5}$ Rebleeding following embolization is high due to interval development of new varices. Treating the portal vein obstruction by venoplasty and stenting was enough to obliterate the jejunal varices surrounding the hepaticojejunostomy in some cases due to effective reduction in the portal hypertension. ${ }^{3}$ Careful selection of the cases is required when there is extension of thrombosis/ occlusion into the intrahepatic portal venous branches. When feasible, a combination of portal venous stenting and embolization of ectopic jejunal varices provides the best line of management. In cases of failure, shunt surgery may be considered as definitive treatment. ${ }^{3}$

\section{Conflict of Interest}

None

\section{References}

1 Lee DH, Kim YH, Lee YJ, et al. CT findings of afferent loop varices after bilioenteric anastomosis in patients with malignant disease. AJR Am J Roentgenol 2013;200(6):1261-1268

2 Taniguchi H, Moriguchi M, Amaike H, Fuji N, Murayama Y, Kosuga T. Hemorrhage from varices in hepaticojejunostomy in the fifth and tenth year after surgery for hepatic hilar bile duct cancer: a case report. Cases J 2008;1(1):59

3 Lee SD, Park S-J, Kim HB, et al. Jejunal varix bleeding with extrahepatic portal vein obstruction after pylorus-preserving pancreatoduodenectomy: report of two cases. Korean J Hepatobiliary Pancreat Surg 2012;16(1):37-42

4 Watson GA, Abu-Shanab A, O'Donohoe RL, Iqbal M. Enteroscopic management of ectopic varices in a patient with liver cirrhosis and portal hypertension. Case Reports Hepatol 2016;2016:2018642

5 Sasamoto A, Kamiya J, Nimura Y, Nagino M. Successful embolization therapy for bleeding from jejunal varices after choledochojejunostomy: report of a case. Surg Today 2010;40(8):788-791 